\title{
Investigating electron beam interactions with nanoparticle capping ligands using correlative liquid phase transmission electron microscopy and fluorescence microscopy
}

Thilini Dissanayake ${ }^{1}$, Mei Wang ${ }^{2}$ and Taylor Woehl ${ }^{2}$

${ }^{1}$ University of Maryland, College Park, Maryland, United States, ${ }^{2}$ University of Maryland, United States

Liquid phase transmission electron microscopy (LPTEM) has been widely used for real time imaging of nanoscale dynamics in nanoparticles (NPs) systems in liquid phase where nanoparticles are functionalized with different types of ligands ranging from small molecules to polymers [1,2,3]. Capping ligands control colloidal interparticle interactions that are important in directing assembly between NPs; however, interactions between the electron beam and capping ligands during LPTEM have not been studied. Radiation chemistry during LPTEM has been extensively studied and utilized in recent years and different types of radicals are produced due to water - electron beam interactions $[4,5]$. In particular, hydroxyl radicals are produced, which rapidly reacts with organic molecules through oxidation reactions [5]. Therefore, it is imperative to investigate the effect of these radicals on surface bound and free ligands to set the dose limits for visualizing nanoparticle assembly processes with minimum electron beam interference.

In this study we applied a correlative LPTEM and fluorescence microscopy (FM) method to examine NPligand-electron beam interactions for a system of silver NPs functionalized with branched polyethylenimine (BPEI). The specific binding of a fluorophore to primary amine functional groups on the BPEI is utilized to investigate electron beam induced BPEI polymerization and degradation on the NPs and silicon nitride $\left(\mathrm{SiN}_{\mathrm{X}}\right)$ membranes during LP-TEM imaging. BPEI functionalized $100 \mathrm{~nm}$ silver NPs were air dried on both the top and bottom membranes of the liquid cell and DI water was flowed into the cell before irradiating different areas of the $\mathrm{SiN}_{\mathrm{X}}$ window of the liquid cell at different dose rates $(0.340$ MGy/s and 0.658 MGy/s) and time intervals (30 s, $5 \mathrm{~min}, 10 \mathrm{~min}$ ) in scanning TEM (STEM) mode. A magnification of 20,000 was used in all LPTEM experiments. After the LPTEM experiment, amide coupling chemistry was employed to label the ligands on the membranes and NPs with carboxyflourescein.

Figure 1 shows a representative FM image showing several different regions of the LPTEM sample irradiated for different times. In these experiments we could see that the sample regions exposed for longer times ( $>5$ mins) had a darker middle with a bright halo around the area, while regions irradiated for short times $(<30$ s) were bright compared to background. We attribute this observation to reactions between $\mathrm{BPEI}$ and electron beam generated hydroxyl radicals occurring at the $\mathrm{SiN}_{\mathrm{x}}$ membrane [6]. At earlier times (<30 s) polymer crosslinking reactions dominate and BPEI ligands crosslink near the $\mathrm{SiN}_{\mathrm{X}}$ membrane giving bright fluorescence. For times $>5$ min polymer chain scission is favored resulting in fragmentation of crosslinked BPEI and diffusion of BPEI fragments to the outer edge of the irradiation area, which crosslink to form the bright halos. To determine whether the nanoparticles had any effect on these reactions, we removed the nanoparticles from the solution through centrifugation and dried the solution of free BPEI ligands on the membranes (Figure 2). Here, we did not observe any difference in the FM images for regions exposed to beam for $30 \mathrm{~s}$ and $1 \mathrm{~min}$, but surprisingly the areas irradiated for $5 \mathrm{mins}$ and 10 mins showed bright fluorescence in contrast to the sample with nanoparticles where dark areas with bright outer edge were observed at these conditions. In a previous study it was shown that the dose 
rate and concentration of radicals is larger near a metal-water interface compared to a $\mathrm{SiN}_{\mathrm{X}}-$ water interface [7]. The silver NPs deposited on the $\mathrm{SiN}_{\mathrm{X}}$ window increased the dose rate near the membrane leading to faster chain scission kinetics whereas without the NPs chain scission kinetics were slower leading the crossliking reactions to dominate [8].
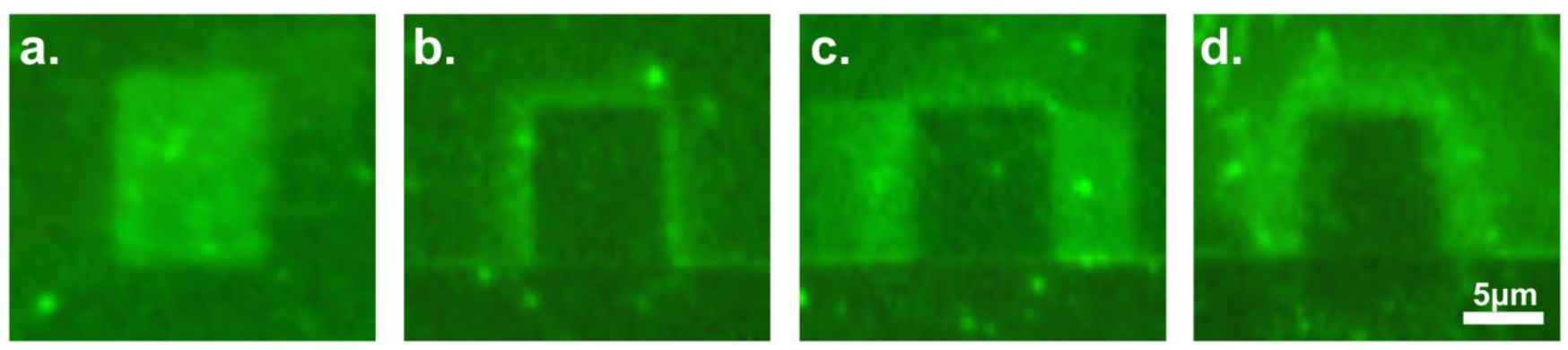

Figure 1. FM image of the $\mathrm{SiN}_{X}$ membrane with NPs after LPTEM imaging. Areas (a) was irradiated at a dose rate of $0.340 \mathrm{MGy} / \mathrm{s}$ and $\mathrm{b}, \mathrm{c}, \mathrm{d}$ were irradiated at a dose rate of $0.658 \mathrm{MGy} / \mathrm{s}$ (total doses: a. $<10.2$ MGy, < 30 s, b. 197 MGy, 5 mins, c. 395 MGy, 10 mins, d. 790 MGy, 20 mins)

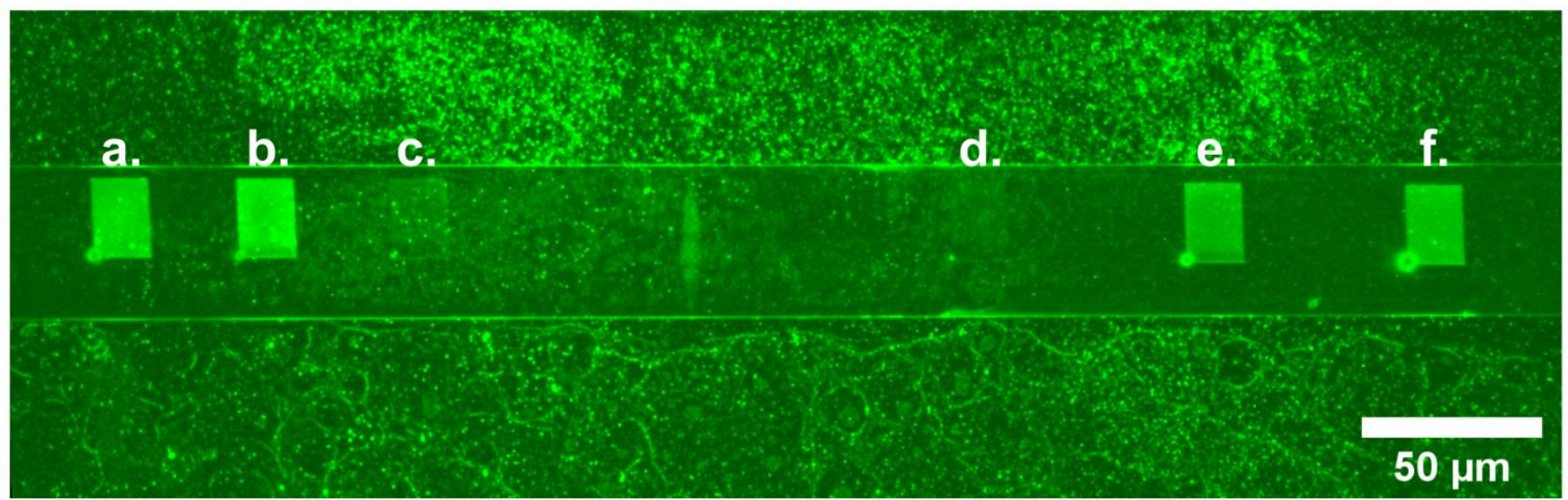

Figure 2. FM image of the $\mathrm{SiN}_{\mathrm{x}}$ membrane without NPs after LPTEM imaging. Areas a, b, c were irradiated at a dose rate of $0.658 \mathrm{MGy} / \mathrm{s}$ and areas d, e, f were irradiated at a dose rate of $0.340 \mathrm{MGy} / \mathrm{s}$. (a. 395 MGy, 10 mins, b. 197 MGy, 5 mins, c. 79 MGy, 1 min, d. 41 MGy, 1 min, e. 102 MGy, 5 mins, f. 204 MGy, 10 mins)

\section{References}

[1] Woehl, T.J. and Prozorov. T. (2015), J. Phys. Chem. C, 119 (36), 21261-21269

[2] Luo, B., Smith, J.W., Ou, Z., Chen, Q. (2017), Acc. Chem. Res., 50 (5), 1125-1133

[3] Jungjohann, K. L., Wheeler, D. R., Polsky, R., Brozik, S. M., Brozik, J. A., \& Rudolph, A. R. (2019), Micron, 119, 54-63

[4] Schneider, N.M., Norton, M.M., Mendel, B.J., Grogan, J.M., Ross, F.M., Bau, H.H. (2014), J. Phys. Chem. C, 118, 38, 22373-22382

[5] Woehl, T.J., and Abellan, P. (2016), J. Microscopy, 265(2), 135-147

[6] Matusiak, M., Kadlubowski, S., Rosiak, J.M. (2020), Radiation Physics \& Chemistry, 169

[7] Gupta, T., Schneider, N. M., Park, J. H., Steingart, D., Ross, F. M. (2018), Nanoscale, 10, 7702

[8] The authors acknowledge funding from the Army Research Office (\#W911NF-20-1-0169). 